\title{
MYositis AsSOcIATED WITH LOCALIZED LIPODYSTROPHY: AN UNRECOGNIZED CONDITION?
}

\author{
H.-J. Gdynia ${ }^{1}$, P. Weydt ${ }^{1}$, A. Ernst ${ }^{2}$, S. Klein ${ }^{2}$, A.-D. Sperfeld ${ }^{1}$, A. Riecker ${ }^{1}$ \\ ${ }^{1}$ Department of Neurology, ${ }^{2}$ Department of Radiology, University of Ulm, Germany
}

\begin{abstract}
Lipodystrophies represent a heterogeneous group of diseases characterized by altered body fat repartition and often metabolic alterations. Here we illustrate a 20 year old male with myositis in association with localized lipodystrophy. Immunohistochemical stainings revealed a regular pattern of dystrophin, dysferlin, sarcoglycans, and theletonin. Furtermore, there was no evidence of Lamin A/C deficiency. A nearly identical clinical and histological picture has been described in three patients up to now. Although it is difficult to speculate on a causative pathophysiological mechanism at this time, it is possible that this association represents an unrecognized condition.
\end{abstract}

Key words: Lipodystrophy; lipoatrophy; myositis; myopathy; muscle biopsy

\section{INTRODUCTION}

Lipoatrophy, also termed lipodystrophy, is a rare condition presenting as a loss of subcutaneous fat. The disorder can occur in generalized, partial, or localized form. Total lipodystrophy can be congenital or acquired and is usually associated with other clinical features such as hyperpigmentation, acanthosis nigricans, diabetes mellitus, glomerulonephritis, and hepatopathy [1]. For further information about inherited lipodystrophy syndromes visit "Online Mendelian Inheritance in Men" at www.ncbi.nlm.nih.gov/Omim. An association with muscle fibre degeneration [2] and dermatomyositis [3, 4] has already been described in the medical literature.

\section{CASE REPORT}

A 20 year old male patient presented with a slowly progressive atrophy of his right buttocks region. Personal history revealed a CK elevation of unknown origin, family history was non-contributory. Physical examination demonstrated a focal atrophy of his proximal right lower extremity, distinctly pronounced in the gluteal region (Fig. 1a). Muscle strength was normal in the whole system; deep-tendon reflexes were unremarkable. Routine laboratory examinations revealed no abnormalities, the creatine-kinase level was normal (88 U/1; Norm < $110 \mathrm{U} / \mathrm{l})$. Laboratory screening for vasculitis was unremarkable. Muscle magnetic resonance imaging revealed a severe subcutaneous and intramuscular lipoatrophy in the clinically affected region (Fig. 1b). Nerve conduction studies were unremarkable. Electromyography showed slight myopathic changes in the right gluteus medius and maximus muscle (s), but a normal pattern in the clinically unaffected musculature (left vastus medialis, right tibialis anterior); there was no evidence for a L5/S1 radiculopathy. A muscle biopsy from the electromyographically affected right gluteus maximus and the unaffected left vastus lateralis muscle was performed. Routine histological stainings included H\&E, modified gomori-trichrome, acid-phosphatase, NAD$\mathrm{H}$, cytochrom-c-oxidase, succinate-dehydrogenase, myoadenylate-desaminase, oil-red-o, perjodacid-schiff, and ATPases. Immuno-histochemical stainings included CD4, CD8, MHC1, MHC2, C5b9, CD68, Dystrophin, Dysferlin, Sarcoglycanes, Lamin A/C, and Theletonin. H\&E staining demonstrated focal endomyseal inflammatory infiltrations in both muscles, pronounced in the right gluteus maximus (Fig. 2 a-c) and to a lesser extent in the left vastus lateralis (Fig. 2 d-f). Immuno-histochemical analysis proved pathological MHC1 expression and a predominance of CD4 over CD8 positive lymphocytes. All other stainings were unremarkable; especially there was no evidence for Lamin A/C deficiency. In summary, the patient showed a localized unilateral lipoatrophy in association with a bilateral myositic process. Therapeutically, oral prednisolone therapy (initially $80 \mathrm{mg} /$ day) was initiated, but without obvious clinical effect after 6 month of treating. One month after corticoid-therapy, a second biopsy from right gluteus maximus muscle was performed for therapy evaluation and to exclude artificial induced inflammatory changes by needle EMG in the first biopsy: In summary, the same histological picture as in the first biopsy was seen, i.e. inflammatory infiltrations in comparable extend. Reapplied muscle magnetic resonance imaging revealed no modification of the lipatrophic process. 

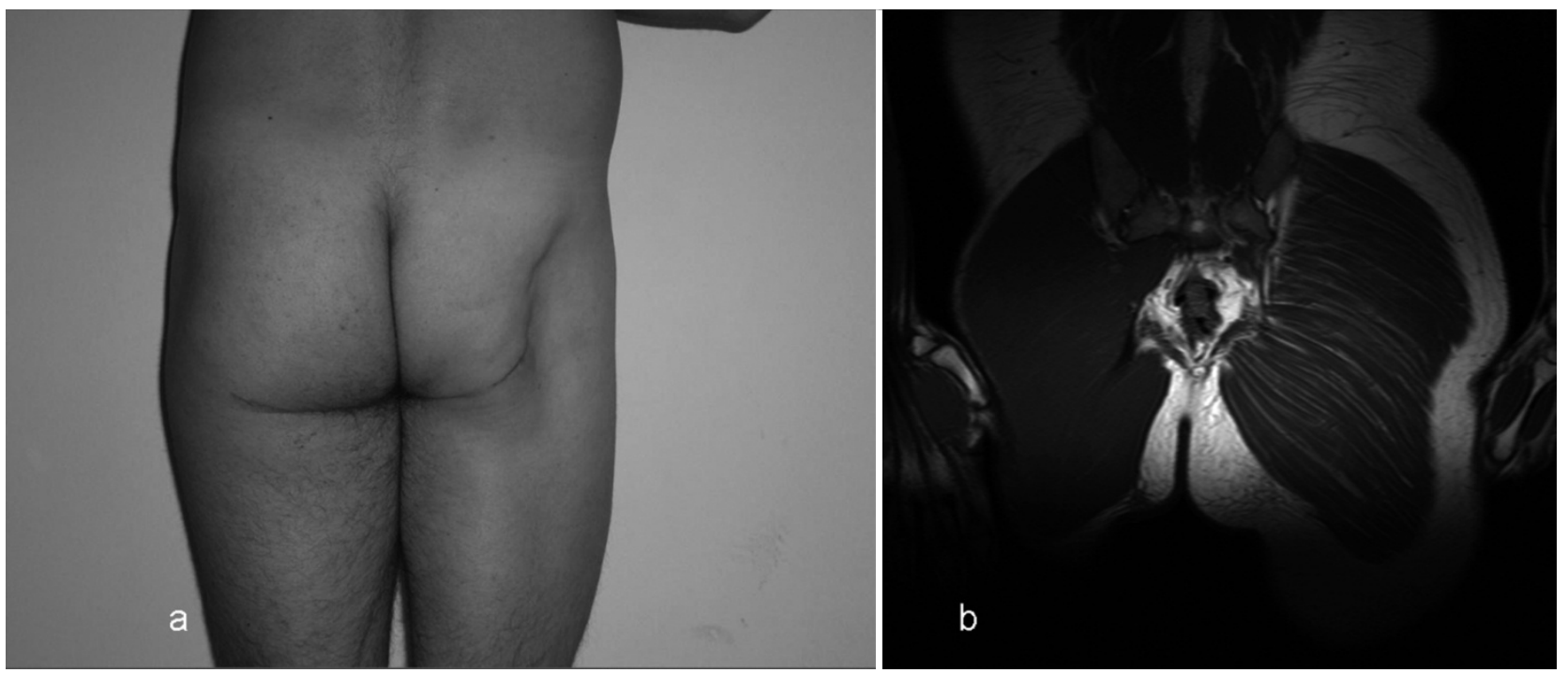

Fig. 1. a: Posterior view of patient demonstrates focal loss of subcutaneous fat overlying the right gluteus muscle. b: Muscle MRI (T2 weighted image) demonstrating severe localized lipoatrophy.
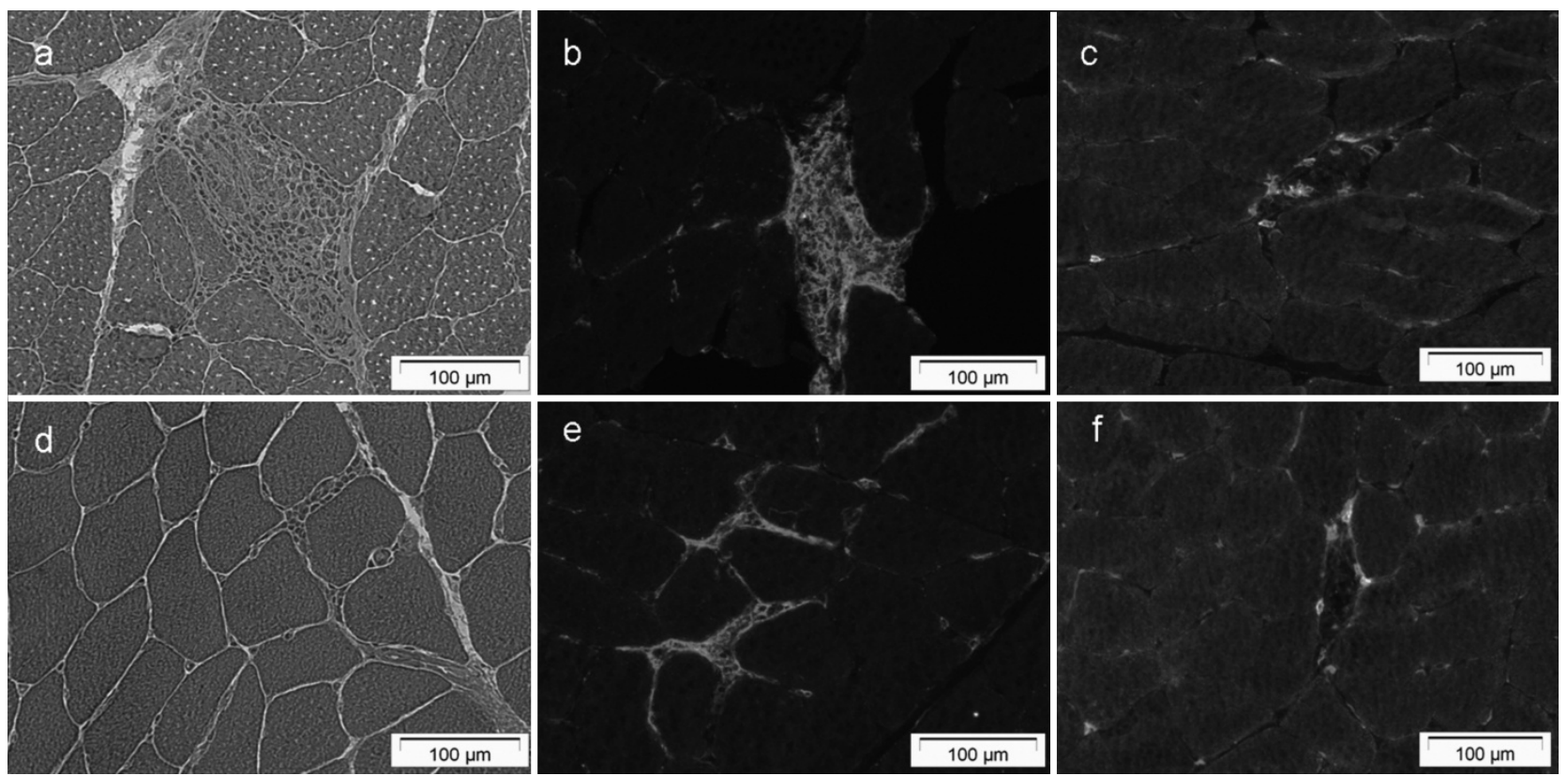

Fig. 2. Skeletal muscle biopsy. a - c: right gluteus maximus (a: Hematoxylin-eosin 1:20; b: CD4 immunostaining 1:20; c: CD8 immunostaining 1:20): Massive endomyseal inflammatory infiltration demonstrated in HE staining. Immunohistochemical analysis showed CD4 positive lymphocytes in the region of the infiltration, but also CD8 positive cells endomyseal. $\mathrm{d}-\mathrm{f}$ : left vastus latealis (d: HE 1:20, e: CD4 1:20; f: CD8 1:20): HE staining revealed a more slightly inflammatory infiltration compared with the findings in the electromyographically affected right gluteus maximus (a), but also a predominance of CD4 positive cells.

\section{DisCUSSION}

To date, histologically confirmed focal or multifocal myositis has been reported in three cases of localized lipoatrophy: In 1982, Palliyath and Garcia described a 54 year old man with a multifocal interstitial myositis associated with localized lipoatrophy isolated in the region of the right buttock [5]. The clinical picture and the histological changes were not progressive over a period of 30 years, so the authors concluded a benign course of this hitherto not described condition. Betteley reported a localized atrophy of the sub- cutaneous fat of the left buttock in a 12 year old boy. In this patient, ipsilateral gluteal muscles were weak, histological examination revealed perivascular mononuclear cell infiltration of the skin. Muscle fibres were atrophic and areas of coagulation necrosis with lymphocyte infiltration were seen [6]. Finally, in 1994, Creange and colleagues [7] described a 24 years old man with severe muscle cramps, fasciculations and atrophy localized to the right lower limb. Computed tomography revealed localized atrophy of the subcutaneous fat. A right quadriceps femoris muscle biopsy showed a pronounced infiltration of the interstitial 
tissue by mononuclear cells, predominating in the walls of small vessels, and around them. Our patient showed a clinical and histological picture nearly identical to these three patients. The patient described by Palliyath and colleague [5] was treated with prednisone $60 \mathrm{mg}$ per day for three weeks without benefit, like our patient. Creange et al. did not treat their patient with steroids [7]. Whereas the patient of Palliyath and colleagues demonstrated mild weakness of the right shoulder girdle musculature, right quadriceps, and gastrocnemius, the muscle strength was clearly not reduced in our patient. Considering the rarity of both disorders (focal myositis and localized lipoatrophy), Creange et al. [7] concluded that this is most likely not a chance association.

\section{CONCLUSIONS}

In our opinion it is difficult to speculate on a causative pathophysiological mechanism of this association, but it seems to be possible that this association represents an unrecognized condition with a benign disease course but absent response to steroid treatment. Due to a psychological strain of the patients, further research is necessary to focus on therapeutically approaches.

\section{REFERENCES}

1. Peters MS, Winkelmann RK. Localised lipoatrophy (atrophic connective tissue disease panniculitis). Arch Dermatol 1980;116:1363-8.

2. Oyanagi K, Sasaki K, Ohama E, Ikuta F, Kawakami A, Miyatani $\mathrm{N}$, et al. An autopsy case of a syndrome with muscular atrophy, decreased subcutaneous fat, skin eruption and hyper g-globulinemia: Peculiar vascular changes and muscle fibre degeneration. Acta Neuropathol (Berl) 1987;73: 313-9.
3. Commens C, O'Neill P, Walker G. Dermatomyositis associated with multifocal lipoatrophy. J Am Acad Dermatol 1990;22:966-9.

4. Kavanagh GM, Colaco B, Kennedy CTC. Juvenile dermatomyositis associated with partial lipoatrophy. J Am Acad Dermatol 1993;28:348-51.

5. Palliyath S, Garcia CA. Multifocal interstitial myositis associated with localised lipoatrophy. Arch Neurol 1982;38: 722-4.

6. Bettley FR. Localised panatrophy. Br J Dermatol 1950;62: 330-1.

7. Creange A, Renard JL, Millet P, Boisnic S, Felten D, Bequet D, Hauw JJ. A patient with one limb interstitial myositis with localised lipoatrophy presenting with severe cramps and fasciculation. J Neurol Neurosurg Psychiatry 1994;57:1541-1543.

Received: May 20, 2008 / Accepted: July 24, 2008

Address for correspondence:

Hans-Jürgen Gdynia M.D.

Department of Neurology, University of Ulm

Muscle Laboratory and Neuromuscular Disorders Unit

Oberer Eselsberg 45

89081 Ulm

Germany

Phone: +49-731-177-5225

Fax: $\quad+49-731-177-1202$

Email: hans-juergen.gdynia@uni-ulm.de 